\title{
$t(15 ; 21)$ translocations leading to the concurrent downregulation of RUNX1 and its transcription factor partner genes SIN3A and TCF12 in myeloid disorders
}

Alberto L'Abbate ${ }^{1 \dagger}$, Doron Tolomeo $^{1 \dagger}$, Francesca De Astis ${ }^{1}$, Angelo Lonoce ${ }^{1}$, Crocifissa Lo Cunsolo ${ }^{2}$, Dominique Mühlematter ${ }^{3}$, Jacqueline Schoumans ${ }^{3}$, Peter Vandenberghe ${ }^{4}$, Achilles Van Hoof ${ }^{5}$, Orazio Palumbo ${ }^{6}$, Massimo Carella ${ }^{6}$, Tommaso Mazza ${ }^{7}$ and Clelia Tiziana Storlazzi ${ }^{{ }^{*}}$

\begin{abstract}
Through a combined approach integrating RNA-Seq, SNP-array, FISH and PCR techniques, we identified two novel $\mathrm{t}(15 ; 21)$ translocations leading to the inactivation of RUNX1 and its partners SIN3A and TCF12. One is a complex $\mathrm{t}(15 ; 21)(\mathrm{q} 24 ; \mathrm{q} 22)$, with both breakpoints mapped the nucleotide level, joining RUNX1 to SIN3A and UBL7-AS1 in a patient with myelodysplasia. The other is a recurrent $\mathrm{t}(15 ; 21)(\mathrm{q} 21 ; \mathrm{q} 22)$, juxtaposing RUNX1 and TCF12, with an opposite transcriptional orientation, in three myeloid leukemia cases. Since our transcriptome analysis indicated a significant number of differentially expressed genes associated with both translocations, we speculate an important pathogenetic role for these alterations involving RUNX1.
\end{abstract}

Keywords: Haploinsufficiency, Tumor suppressor genes, AML, MDS

\section{Main text}

Translocations involving $R U N X 1$ are known to decrease the function of the encoded protein in myelodysplastic syndromes (MDS) and acute myeloid leukemia (AML) [1]. For those involving chromosome $15, S V 2 B$ was the only RUNX1 partner gene identified in AML [2].

We report on two novel $t(15 ; 21)$ alterations leading to the concurrent disruption of RUNX1 and SIN3A or TCF12 (Additional file 1: Table S1). Another interrupted gene is the UBL7-AS1 long noncoding RNA gene.

In case 1, FISH experiments (Additional file 2: Table S2) mapped the breakpoint on $\operatorname{der}(21)$ within intron 7 of RUNX1 (Additional file 3: Figure S1A-C). Moreover, SNP array analysis identified a 908-kb deletion near the $15 \mathrm{q}$ breakpoint on der(15) (Additional file 4: Table S3 and Additional file 3: Figure S1D-F). Genomic PCR revealed that intron 7 of RUNX1 (chr21:36194775) was joined at intron 3 of SIN3A (chr15:75708434) (Fig. 1a and

\footnotetext{
*Correspondence: cleliatiziana.storlazzi@uniba.it

${ }^{\dagger}$ Equal contributors

'Department of Biology, University of Bari, Bari, Italy

Full list of author information is available at the end of the article
}

Additional file 5: Table S4) on der(21). Furthermore, intron 7 of RUNX1 (chr21:36194861) was fused to the inverted sequence of $U B L 7$ (NM_032907.4) at intron 1 (chr15:74751664) on der(15), suggesting that a submicroscopic inversion accompanying the translocation led to the juxtaposition of RUNX1 and UBL7-AS1 with the same transcriptional orientation (Fig. 1a). ChimeraScan analysis of RNA-Seq data identified the fusion of SIN3A (exon 3; NM_015477) to RUNX1 (exon 8; NM_001754) and of RUNX1 (exon 7; NM_001754) to UBL7-AS1 (intron 1; NR_038449.1). Both chimeric transcripts were validated by RT-PCR (Fig. 1b and Additional file 6: Table S5). In silico translation of the in-frame $5^{\prime}-\operatorname{SIN} 3 A / 3^{\prime}-R U N X 1$ showed two ORFs of 171 and 163 amino acids, respectively, the first one retaining the transactivation domain of RUNX1 (Fig. 1c). The out-of-frame 5'-RUNX1/3'UBL7-AS1 encoded a protein of 373 amino acids, showing the substitution of RunxI with a GVQW putative binding domain (Fig. 1d). Thus, both chimeric SIN3A/RUNX1 and RUNX1/UBL7-AS1 encoded for truncated SIN3A and RUNX1 proteins. Interestingly, RT-qPCR revealed that the full-length RUNX1 (NM_001754) and the 3' portion of 


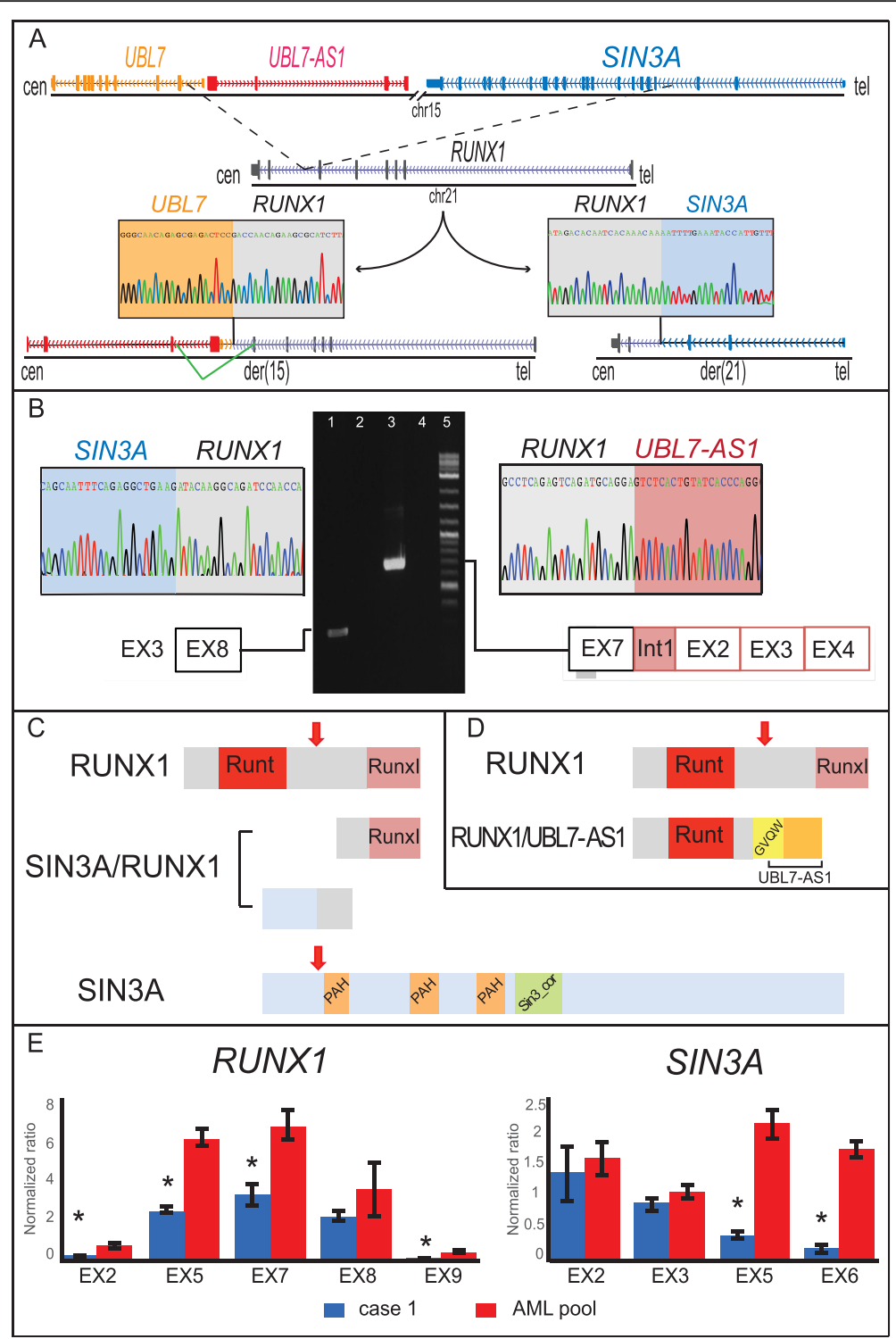

Fig. $1 \mathrm{t}(15 ; 21)(\mathrm{q} 24 ; \mathrm{q} 22)$ translocation in case 1. a Genomic breakpoints on chromosomes 15 and 21 in case 1: Top, schematic representation of wild-type chromosomes (black lines) and involved genes (exons are shown as rectangles and introns as lines connecting exons, with arrowheads indicating the direction of transcription). The dashed black lines indicate the breakpoints within all genes. Bottom, partial chromatograms indicate fusion sequences on der(15) (left) [GenBank: KT336107] and der(21) (right) [GenBank: KT336106]. The splicing event creating the runt-related transcription factor 1 (RUNX1)/UBL7-AS1 fusion transcript is indicated in green. $\mathbf{b}$ RUNX1 fusion transcripts: reverse transcription polymerase chain reaction (RT-PCR) products corresponding to SIN3A/RUNX1 [GenBank: KT336104] and RUNX1/UBL7-AS1 [GenBank: KT336105] fusion transcripts (lanes 1 and 3, respectively) in case 1 are shown in the middle of the panel. Lanes 2 and 4: negative normal bone marrow samples. Lane 5: 2-log DNA ladder (New England Biolabs, Milan, Italy). Partial chromatograms (top) and structure (bottom) of SIN3A/RUNX1 and RUNX1/UBL7-AS1 PCR productS are on the left and on the right, respectively. $\mathbf{c}, \mathbf{d}$ RUNX1 chimeric proteins: both panels show in silico translation (ORFfinder and BlastP) of both wild-type and chimeric RUNX1 and SIN3A proteins. Arrows indicate the truncation breakpoints of wild-type proteins. e Evaluation of RUNX1 and SIN3A expression levels in case 1: exon-specific reverse transcription quantitative PCR analysis of RUNX1 (left) and SIN3A (right) was performed in case 1 vs a control pooled sample of patients with acute myeloid leukemia. Asterisks indicate statistically significant results $(P<.05)$

SIN3A (NM_015477) were downregulated (Fig. 1e), whereas $U B L 7-A S 1$ was overexpressed (Additional file 7: Figure S2). Further, we evaluated the molecular impact of these fusion transcripts through a differential expression analysis, using the control datasets of $19 \mathrm{AML}$ cases with normal karyotype from the The Cancer Genome
Atlas data bank (http://cancergenome.nih.gov/). The results obtained by the QIAGEN's Ingenuity Pathway Analysis (IPA ${ }^{\circ}$, QIAGEN Redwood City) [3], following the Partek Genomics Suite 6.6 (Partek Inc., St. Louis, MO, USA), indicated a significant number of differentially expressed genes within the AML pathway. The cell proliferation 
and myeloid differentiation pathways were significantly activated, whereas apoptosis exhibited reduced activity (Additional file 8: Figure S3 and Additional file 9: Table S6).

The results obtained in case 1 clearly suggest a role as a tumor suppressor (TS) gene not only for RUNX1 (the shorter RUNX1 encoded by the chimeric RUNX1/UBL7$A S 1$ should behave as a dominant negative mutant of the wild-type RUNX1), but also for SIN3A. We speculate that the inactivation of both proteins should have led to an abnormal activation of RUNX1/SIN3A target genes, leading to myelodysplasia. Even if haploinsufficiency was never reported for SIN3A, its role as a TS has been described in other tumors [4]. Notably, the SIN3A corepressor was known to interact with RUNX1 [5], leading to the transcriptional inactivation of their target genes [6].

In cases 2 and 3, FISH indicated that RUNX1 was interrupted within intron 7. Additionally, in case 4, a 600$\mathrm{kb}$ deletion removed the $5^{\prime}$ portion of RUNX1 starting from intron 6 (Fig. 2a and Additional file 1: Table S1). In all cases, RUNX1 was joined with an opposite transcriptional orientation to intron 3 of TCF12 (NM_003205), a basic helix-loop-helix transcription factor (Fig. 2b) fused with $M L L$ in MDS [7] and recurrently mutated in myeloproliferative disorders [8]. Notably, in case 2, RT-qPCR indicated the downregulation of both RUNX1 and TCF12 (Fig. 2c) and IPA analysis disclosed significant deregulation of the AML pathway. The number of altered pathways was slightly higher than in case 1 (146 vs. 136) and mostly overlapped the previously described categories. Particularly, RUNX1 was shown to control many differentially expressed genes involved in cell cycle regulation, inflammatory response, and transcription regulation (Additional file 8: Figure S3 and Additional file 9: Table S6).

We thus suggest a TS role for TCF12 in myeloid disorders, as already described in colon carcinoma [9]. The concurrent inactivation of RUNX1 and TCF12 should mimic the same leukemogenic effect of the $t(8 ; 21)$ RUNX1/CBFA2T1 fusion protein. E proteins, like TCF12,
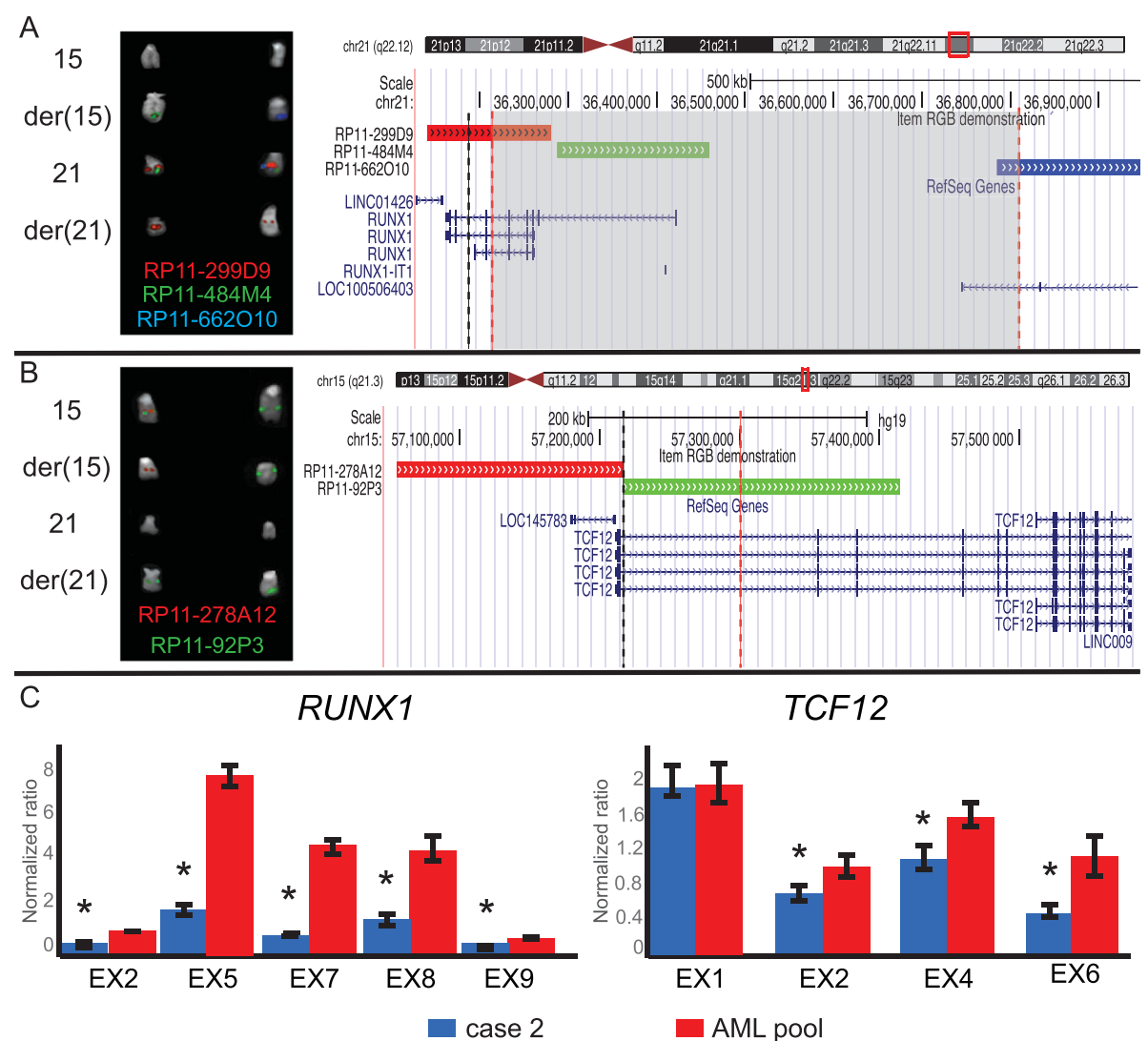

TCF12

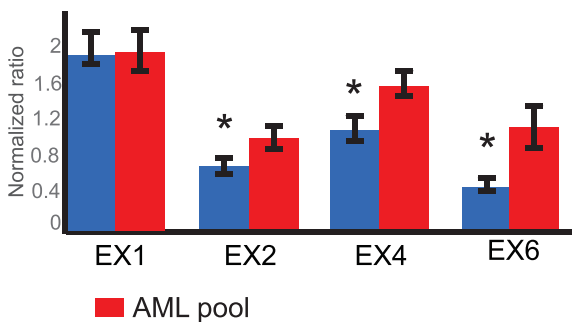

Fig. 2 t(15;21)(q21;q22) translocation in cases 2, 3, and 4. a, b Breakpoints on chromosomes 21 and 15: partial karyotypes showing fluorescence in situ hybridization (FISH) results that allowed the mapping of $t(15 ; 21)$ translocation breakpoints on der(21) (a) and der(15) (b), using the consistently colored probes listed for cases 2 (first column) and 4 (second column). RP11-662010 was used only in case 4 . Cases 2 and 3 shared the same breakpoints (data not shown). On the right, the map of the BAC probes used in the FISH experiments, according to GRCh37/hg19, and identifying both translocation and deletion breakpoints, is shown. The black and orange dashed lines indicate the breakpoints in cases 2 and 4 , respectively. The grey rectangle encompasses the deleted region flanking the translocation breakpoint in case 4. c Evaluation of RUNX1 and TCF12 expression levels: exon-specific reverse transcription quantitative polymerase chain reaction results of runt-related transcription factor 1 (RUNX1; left) and transcription factor 12 (TCF12; right) in case 2 . Asterisks indicate statistically significant results $(P<.05)$ 
are inactivated through their interaction with the domain TAFH of CBFA2T1, leading to the inhibition of the p300/ CBP histone acetyltransferase recruitment at their target genes' promoters and consequently to the lack of activation of genes with E-box promoters [10].

To summarize, we here identified three novel RUNX1 partner genes, including two transcription factors and a long noncoding RNA, in $2 \mathrm{t}(15 ; 21)$ translocations. Both of the $\mathrm{t}(15 ; 21)$ translocations resulted in the concurrent inactivation of RUNX1 and one related transcription factor (SIN3A or TCF12), leading to the potential haploinsufficiency of both involved genes. Moreover, the IPA analyses clearly indicated that the AML pathway was significantly deregulated in our samples, and showed that RUNX1, SIN3A, or TCF12 have a crucial impact on differentially expressed genes. The analysis of additional cases harboring $\mathrm{t}(15 ; 21)$ translocations will be helpful to better understand the pathogenetic impact of these alterations in myeloid neoplasms.

\section{Ethics approval}

This study was performed in agreement with the Declaration of Helsinki, and approved by the Ethical Committee at the "S. Martino" hospital, Belluno (Italy); Centre Hospitalier Universitaire Vaudois CHUV, Lausanne (Switzerland); University Hospital Leuven, (Belgium).

\section{Consent for publication}

Written informed consent was obtained from the patients for publication of this letter.

\section{Additional files}

Additional file 1: Table S1. Cytogenetic and clinical features of the patients included in the study. (DOCX $8 \mathrm{~kb}$ )

Additional file 2: Table S2. FISH results obtained with BAC and fosmid probes in all the cases included in the study. (DOCX $125 \mathrm{~kb}$ )

Additional file 3: Figure S1. FISH mapping of the $t(15 ; 21)$ translocation breakpoints in case 1. (A, B) Merged pseudocolored fluorescence in situ hybridization (FISH) results obtained with consistently colored BAC and fosmid probes spanning runt-related transcription factor 1. (C) Map of the probes used in the FISH experiments in panels A and B. The black dashed line indicates the breakpoint within the gene. (D, E) FISH results obtained with BAC and fosmid probes used to map the proximal (D) and distal breakpoints (E) on chromosome 15. (F) Map of the probes used in the FISH experiments, identifying both translocation and deletions breakpoints. Black dashed lines indicate the breakpoints; the grey rectangle encompasses the deleted region flanking the translocation breakpoint. (EPS $3237 \mathrm{~kb}$ )

Additional file 4: Table S3. SNP array results [EMBL: E-MTAB-3782] obtained in case 1. (DOCX $123 \mathrm{~kb}$ )

Additional file 5: Table S4. List of primers used for RT-PCR, RT-qPCR and Sanger sequencing. (DOC $91 \mathrm{~kb}$ )

Additional file 6: Table S5. Case 1 Chimerascan output: in bold the fusion genes validated by RT-PCR and Sanger sequencing. Case 2 Chimerascan output. (XLSX $309 \mathrm{~kb}$ )

Additional file 7: Figure S2. Expression pattern analysis of UBL7-AS1 in case 1. Exon-specific reverse transcription quantitative polymerase chain reaction analysis of UBL7-AS1 in case 1 vs a control pooled sample of patients with acute myeloid leukemia. Asterisks indicate statistically significant results $(P<.05)$. (EPS $246 \mathrm{~kb})$

Additional file 8: Figure S3. The IPA acute myeloid leukemia signaling pathway for cases 1 and 2. Orange and blue glyphs indicate genes with predicted enhanced or reduced activity, respectively. Green symbols represent downregulated genes. Equally, orange and blue toothed wheels stand for activated or inhibited biological processes. Case 1 differs from case 2 for its overall activation z-scores (3.5 vs 2.5). IPA, Ingenuity pathway analysis. (JPG $3319 \mathrm{~kb}$ )

Additional file 9: Table S6. IPA analysis results obtained in case 1. IPA analysis results obtained in case 2 . (XLS $232 \mathrm{~kb}$ )

\section{Abbreviations}

AML: acute myeloid leukemia; FISH: fluorescence in situ hybridization; IPA: Ingenuity pathway analysis; MDS: myelodysplastic syndromes; PCR: polymerase chain reaction; RT-PCR: reverse transcription polymerase chain reaction; RT-qPCR: reverse transcription quantitative polymerase chain reaction.

\section{Competing interests}

The authors declare that they have no competing interests.

\section{Authors' contributions}

ALA performed bioinformatics analysis, analyzed all data, and wrote the paper; DT performed FISH analyses, molecular experiments and wrote the paper; FDA and AL performed FISH analyses; CLC, DM, JS, PV, and AVH prepared samples for FISH and molecular analyses, and provided clinical data of the patients; OP and MC performed SNP array CGH analysis; TM performed bioinformatics analysis; CTS reviewed all the data, and wrote the manuscript. All authors read and approved the final manuscript.

Funding

This work was supported by Associazione Italiana Ricerca sul Cancro (AIRC MFAG2011 No.11405 to CTS).

\section{Author details}

${ }^{1}$ Department of Biology, University of Bari, Bari, Italy. ${ }^{2} \cup O$ Anatomia Patologica, Ospedale S. Martino, Belluno, Italy. ${ }^{3}$ Unité de génétique du cancer, Service de génétique médicale, Centre Hospitalier Universitaire Vaudois CHUV, Lausanne, Switzerland. ${ }^{4}$ Center for Human Genetics and Department of Hematology, University Hospital Leuven and KU Leuven, Leuven, Belgium. ${ }^{5}$ Department of Haematology, AZ Sint-Jan AV, Brugge, Belgium. ${ }^{6}$ Medical Genetics Unit, IRCCS Casa Sollievo della Sofferenza Hospital, San Giovanni Rotondo, Italy. ${ }^{7}$ IRCCS Casa Sollievo della Sofferenza, Mendel Institute, San Giovanni Rotondo, Italy.

Received: 16 October 2015 Accepted: 11 December 2015

Published online: 16 December 2015

\section{References}

1. De Braekeleer E, Douet-Guilbert N, Morel F, Le Bris MJ, Ferec C, De Braekeleer M. RUNX1 translocations and fusion genes in malignant hemopathies. Future Oncol. 2011;7:77-91.

2. Giguere A, Hebert J. Identification of a novel fusion gene involving RUNX and the antisense strand of SV2B in a BCR-ABL1-positive acute leukemia. Genes Chromosomes Cancer. 2013;52:1114-22.

3. Ingenuity Pathway Analysis IPA ${ }^{\oplus} \mathrm{QRC}$. http://www.qiagen.com/ingenuity.

4. Suzuki H, Ouchida M, Yamamoto H, Yano M, Toyooka S, Aoe M, et al. Decreased expression of the SIN3A gene, a candidate tumor suppressor located at the prevalent allelic loss region 15q23 in non-small cell lung cancer. Lung Cancer. 2008:59:24-31.

5. Hu Z, Gu X, Baraoidan K, Ibanez V, Sharma A, Kadkol S, et al. RUNX1 regulates corepressor interactions of PU.1. Blood. 2011;117:6498-508.

6. Lutterbach B, Westendorf JJ, Linggi B, Isaac S, Seto E, Hiebert SW. A mechanism of repression by acute myeloid leukemia-1, the target of multiple chromosomal translocations in acute leukemia. J Biol Chem. 2000;275:651-6.

7. Meyer C, Kowarz E, Yip SF, Wan TS, Chan TK, Dingermann T, et al. A complex MLL rearrangement identified five years after initial MDS diagnosis results in out-of-frame fusions without progression to acute leukemia. Cancer Genet. 2011;204:557-62. 
8. Tenedini E, Bernardis I, Artusi V, Artuso L, Roncaglia E, Guglielmelli P, et al. Targeted cancer exome sequencing reveals recurrent mutations in myeloproliferative neoplasms. Leukemia. 2014;28:1052-9.

9. Starr TK, Allaei R, Silverstein KA, Staggs RA, Sarver AL, Bergemann TL, et al. A transposon-based genetic screen in mice identifies genes altered in colorectal cancer. Science. 2009;323:1747-50.

10. Park S, Chen W, Cierpicki T, Tonelli M, Cai X, Speck NA, et al. Structure of the AML1-ETO ETAFH domain-HEB peptide complex and its contribution to AML1-ETO activity. Blood. 2009;113:3558-67.

Submit your next manuscript to BioMed Central and we will help you at every step:

- We accept pre-submission inquiries

- Our selector tool helps you to find the most relevant journal

- We provide round the clock customer support

- Convenient online submission

- Thorough peer review

- Inclusion in PubMed and all major indexing services

- Maximum visibility for your research

Submit your manuscript at www.biomedcentral.com/submit 